Archives de sciences sociales des religions

$177 \mid 2017$

Mondes juifs en mouvement

\title{
In Memoriam François-André Isambert (1924-2017)
}

\section{Pierre Lassave}

\section{(2) OpenEdition}

Journals

Édition électronique

URL : http://journals.openedition.org/assr/29235

DOI : 10.4000/assr.29235

ISSN : $1777-5825$

Éditeur

Éditions de l'EHESS

Édition imprimée

Date de publication : 1 mars 2017

Pagination : 9-13

ISSN : 0335-5985

\section{Référence électronique}

Pierre Lassave, «In Memoriam François-André Isambert (1924-2017) 》, Archives de sciences sociales des religions [En ligne], 177 | 2017, mis en ligne le 01 mars 2017, consulté le 06 janvier 2021. URL : http://journals.openedition.org/assr/29235 ; DOI : https://doi.org/10.4000/assr.29235 


\section{In Memoriam François-André Isambert (1924-2017)}

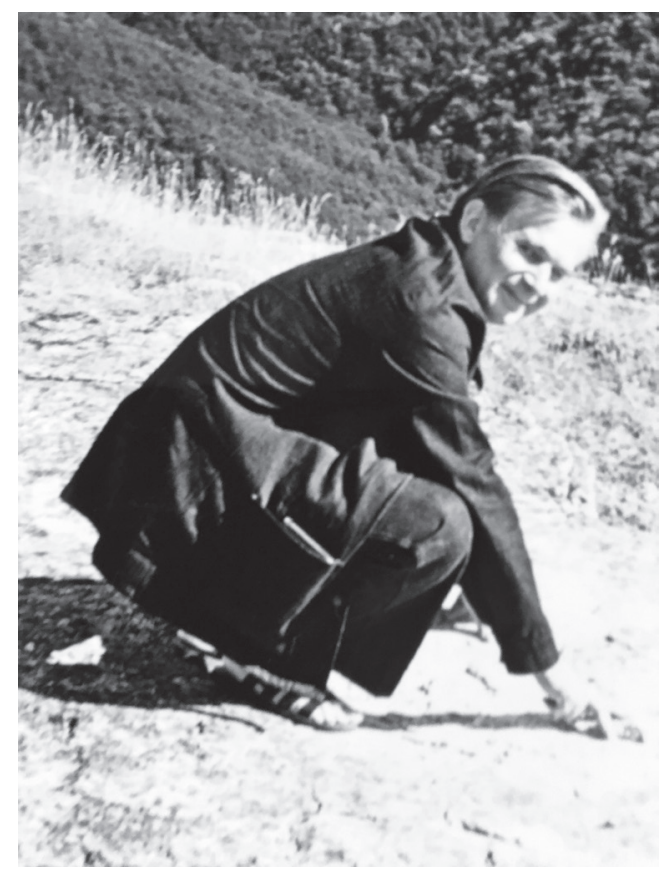

Il y a seulement deux ans, nos colonnes (n 168) accueillaient un dernier texte de François-André Isambert dans lequel il présentait le manuscrit inédit de son ancêtre homonyme qui était une longue étude, datée de 1843, d'exégèse historique du Nouveau Testament dont une des hypothèses était que Jésus aurait vécu bien plus longtemps que ce qu'en dit la tradition. La présentation de ce manuscrit consultable à la Bibliothèque nationale était signée non sans une pointe d'humour à l'âge de quatre-vingt-dix ans, par «François-André 
Isambert Jr ». Notre junior vient de disparaître, tirant le dernier le rideau de la scène primitive des six fondateurs de notre revue. Une nouvelle page s'ouvre dorénavant, celle du travail de mémoire et d'histoire sur ce que leur œuvre a apporté à la connaissance des faits religieux et plus largement de la société, tant pour hier que pour aujourd'hui, présent et passé s'éclairant réciproquement.

François-André Isambert est né à Coblence en 1924 où son père assurait alors le secrétariat de la Commission interalliée pour le territoire de la Rhénanie. Après ses études à Paris (Janson-de-Sailly) puis à Lyon pendant l'Occupation, l'étudiant de khâgne embrasse la cause de la Résistance aux côtés des réseaux d'étudiants chrétiens. Il s'engage en 1944 dans la Première Armée qui libère l'Alsace. Agrégé de philosophie en 1947, il enseigne à Besançon, marié avec Viviane Jamati, future sociologue du travail féminin puis de l'éducation. Comme nombre de philosophes engagés dans le mouvement de l'histoire, Isambert se tourne vers la sociologie et devient assistant de Georges Gurvitch à la Sorbonne. Préoccupé par le fossé entre le monde ouvrier et le christianisme, il se rapproche de Gabriel Le Bras qui le recrute au Centre d'études sociologiques du CNRS dans le Groupe de sociologie des religions créé en 1954 avec Henri Desroche et qui est comme l'on sait à l'origine de notre revue.

Son premier essai, Christianisme et classe ouvrière (1961) prend la mesure statistique des écarts tout en apportant par l'histoire des nuances au schéma de la déchristianisation du monde. Dans ses travaux de mathématique sociale, Raymond Boudon a pu parler du «modèle écologique d'Isambert » consistant à montrer par des corrélations statistiques sophistiquées que plus l'ouvrier habite un quartier ouvrier plus grande est sa distance à l'Église. En parallèle, et après d'autres historiens, comme son grand-père Gaston auteur d'une histoire remarquée des idées socialistes, notre jeune chercheur est parti fouiller dans la mémoire des utopies chrétiennes du mouvement ouvrier.

Sa thèse d'État sous la direction de Gurvitch puis de Raymond Aron, De la charbonnerie au saint-simonisme. Étude sur la jeunesse de Buchez (1966) et Buchez ou l'âge théologique de la sociologie (1967), ouvre ainsi, à travers la figure méconnue de Philippe Buchez, une fenêtre sur les liens de parenté entre le catholicisme, le socialisme et la science du social à l'âge industriel. Sa thèse complémentaire sur la sociologie des fêtes de Noël et du Nouvel An, sous la direction de Jean Stoetzel, marque la diversification de ses centres d'intérêt et de ses méthodes d'enquête tant quantitatives que qualitatives.

Dans les années 1960, Isambert rejoint l'enseignement supérieur, professeur à Lille puis à Nanterre. Membre de la Revue française de sociologie, aux côtés de Stoetzel son fondateur, l'enseignant-chercheur participe activement à la reconnaissance universitaire de la discipline. En 1971, il est élu directeur d'études à la VIe section de l'EPHE - bientôt EHESS en 1975 - poursuivant son ouverture à la sociologie des religions inaugurée par Le Bras. Ses travaux sur la «sécularisation interne» et sur la «religion populaire» le conduisent au centre de débats savants, tant au sein de l'Association française de sociologie des religions (AFSR) qu'il contribue à déconfessionnaliser avec Jacques 
Maître, qu'au sein des conférences internationales en pleine extension (CISR). Sa sagacité critique montre comment ces constructions intellectuelles à forte charge polémique relèvent de fractures qui traversent conjointement les institutions ecclésiastiques et académiques. Revenant plus fondamentalement aux thèses de l'école durkheimienne sur la nature sociale du sacré, il décrypte par ailleurs les mécanismes de l'efficacité symbolique des rituels transformés, notamment après les réformes de Vatican II, ce qui aboutit à la publication de deux essais remarqués: Rite et efficacité symbolique. Essai d'anthropologie sociologique (1979) et Le sens du sacré. Fête et religion populaire (1982). Analyste de la force agissante, contagieuse et ambivalente du sacré, il atteint le seuil des mécanismes psychophysiologiques entrevus par Claude Lévi-Strauss à propos de la cure chamanique, mais sans aller au-delà de la fenêtre qu'il ouvre sur une pragmatique de la liturgie en prenant appui sur la théorie des actes de langage de John L. Austin et John R. Searle.

Observateur attentif des «situations à propositions profuses », comme il le dit à propos des débats sur la contraception et l'avortement, il créée à partir des années 1980 un laboratoire de sociologie de l'éthique avec ses collaborateurs Jean-Paul Terrenoire et Paul Ladrière. Ce centre qui reprend à nouveaux frais le projet de Gurvitch d'une sociologie de la morale, s'adonne entre autres à l'étude des justifications et des litiges qui accompagnent tout processus biomédical engageant le corps, la vie et la mort: manipulations génétiques, don d'organes, assistance à la fin de vie, etc. La généalogie de la bioéthique qu'il entreprend avec ses correspondants américains (Renée C. Fox) l'amène à mettre au jour le rapport expérimental à l'humain au même titre que Michel Foucault avait exploré la dimension clinique de la médecine. La phénoménologie de l'action théorisée par Alfred Schütz et la rationalité en valeur définie par Max Weber lui servent de balises. C'est à ce moment exploratoire, dans un perspectivisme sociologique résolu qu'il épingle dans une note critique très lue de la Revue française de sociologie (1985) le "programme fort» d'anthropologie des sciences de David Bloor, pris selon lui «en porte-à-faux entre une critique épistémologique qui se défend contre le réalisme scientifique et une sociologie qui développe les caractères d'une nature sociale confondue avec une procédure explicative de type mécaniste».

Dans son livre bilan qui rassemble nombre de ses articles les plus commentés, De la religion à l'éthique (1992), il vise moins un improbable remplacement du religieux par l'éthique qu'une attention renouvelée aux références métaphysiques investies dans le débat public sur le statut du vivant. En cela sa pensée riche en nuances nous prévient contre tout étonnement face aux résurgences passionnelles d'aujourd'hui au nom de la "post-vérité ». Peu à l'aise dans les tâches croissantes d'organisation de la recherche malgré diverses responsabilités prises au plus haut niveau dans les comités nationaux, il a cependant formé quelques chercheurs qui se réclament de son héritage exigeant, notamment Patrick Pharo, spécialiste des questions morales, et Simone Bateman, analyste des pratiques biomédicales à controverses, sans parler des sociologues des religions qui telle Danièle Hervieu-Léger se réfèrent à lui. 
Ses derniers travaux d'épistémologie critique qui reviennent sur la réduction des sciences à leur socialité, sur les traductions fautives de Max Weber, sur les premières enquêtes de Paul Lazarsfeld sur les chômeurs de Marienthal dans l'Autriche des années 1930 ou sur le coefficient moral de la théorie économique de John Maynard Keynes, témoignent de la curiosité universelle de cet auteur. Compagnon de route d'Alain Touraine, de Raymond Boudon et de Pierre Bourdieu, son œuvre demeure moins connue des manuels académiques et du grand public que celle de ces derniers. Mais comme on vient de le suggérer, nombre de ses articles et de ses essais ont été pris comme modèles de solidité théorique et de finesse d'analyse par au moins deux générations de sociologues. Avec sa disparition, le devoir d'inventaire frappe à la porte ${ }^{1}$.

\section{$\because$}

De façon plus personnelle, et pour tous ceux qui n'ont pu assister aux obsèques familiales et civiles, voici les paroles qu'il m'a été donné de prononcer au cimetière d'Orgeval, le vendredi 24 février 2017:

François,

Il y a cinq ans seulement, je t'ai cherché sur la toile et suis tombé sur ton ancêtre homonyme, François-André Isambert, juriste et député connu pour son combat en faveur de l'abolition de l'esclavage sous la Monarchie de Juillet. Pour moi, Isambert c'était pourtant d'abord le nom d'un professeur discret mais à la critique sûre. Que ce soit pour chiffrer et comprendre le fossé entre le monde ouvrier et le christianisme, pour décrypter les valeurs morales engagées dans les débats autour de l'avortement, ou pour prévenir des risques de réduire toute science à ses conditions sociales.

Ton nom a longtemps flotté dans ma mémoire comme une référence savante avant que sur le tard les sinuosités de ma trajectoire me conduisent sur les traces de cet étrange Groupe de sociologie des religions d'après-guerre dont tu as été l'un des membres fondateurs aux côtés de Gabriel Le Bras, Henri Desroche, Émile Poulat, Jacques Maître et Jean Séguy. Une équipe peu conformiste qui a fait son trou et a assuré sa postérité, quand bien même la sociologie des religions a longtemps été une spécialité assez marginale dans ce pays. L'active revue Archives de sciences sociales des religions que tu as contribué à lancer dans les années cinquante est aujourd'hui florissante.

Il y a cinq ans seulement, je t'ai rencontré à Montrouge car tu cherchais à faire connaître le manuscrit de ton ancêtre homonyme, une étude historique inédite sur la Vie de Jésus qui anticipait sur celle d'Ernest Renan. J'ai alors profité de ta demande pour en savoir plus sur ta propre histoire. Ainsi ai-je également rencontré Viviane, devenue chère à mon cour. Tous les deux,

1. Une bibliographie complète, accompagnée d'éléments de parcours, est consultable sur notre site: http://assr.revues.org/25703 
vous avez su me parler des heurs et malheurs d'une existence communément consacrée à la connaissance du monde social.

C'est dans une clinique de repos que, cher François, nous sommes passés du Vous au Tu. Bien que fort réticent à me raconter ta vie et cherchant péniblement tes mots, tu m'attendais dans le couloir certains jours de l'été 2014 en me reprochant d'arriver avec une minute de retard. Je ne réparais pas seulement ton appareil auditif ou ton téléphone portable, mais je te faisais aussi sortir par la parole de la prison où tu étais: "Aujourd'hui, on s'est bien rincé le cerveau, hein! Et si on passait du Vous au Tu? ", m'as-tu dit un jour avec un éclair dans le regard. Cet éclair de l'esprit parcourt ta vie et ton ouvre. La ferveur et l'ardeur aussi, termes dont tu me détaillais les nuances. Je n'ai pas eu le temps ni le courage d'évoquer avec toi la fureur et la frayeur qui t'ont également trop de fois traversé et paralysé.

L'engagement dans la Résistance, tout jeune étudiant chrétien. La déclaration définitive d'amour à Viviane. La réflexion sur l'être et le néant après la mort soudaine d'un camarade de combat fauché à tes pieds par une mitrailleuse sur le front d'Alsace. La conversion de l'agrégé de philosophie à la sociologie renaissante. La joie des premiers sourires d'Emmanuel, de Françoise et de Christiane. L'investissement acharné dans les corrélations linéaires entre la structure sociale et la pratique religieuse, dans l'histoire des précurseurs de la pensée sociale, dans l'anthropologie des rites, des sacrements et des fêtes. Puis, au milieu du gué, le rebond vers la généalogie de la bioéthique, en passant par les États-Unis. Ta matière était des "situations à propositions profuses ", comme tu disais, autour des questions posées par la vie interrompue, transplantée, manipulée, augmentée. L'observation du monde n'est jamais allée pour toi sans réflexion sur le sens de ce qui se passe et de ce qui nous fait. Descartes, Kant, Husserl et surtout Durkheim et Weber, inlassablement revisités.

Tu ne nous laisses pas pour finir qu'une exigence d'élucider la complexité des choses mais aussi un univers caché d'émotions et de sentiments les plus vifs qu'une pointe d'humour laissait souvent entrevoir. Moïse sauvé des eaux montantes du Rhin, à Coblence ta ville natale, c'est aussi toi au berceau que tes parents évacuaient par précaution. La motte de terre glaise dans laquelle tu creusais des boyaux pour y faire circuler des billes, c'était ton jeu inventé dans le jardin de ta maison bleue de l'enfance, ici à Orgeval, lieu de villégiature de tes parents où tu as décidé de retourner pour toujours. La prochaine fois que je passe par mon midi natal, j'irai me pointer comme toi sur le cap de la Gorguette à Sanary-sur-Mer, scruter au loin La Ciotat et son île verte près de laquelle j'ai appris tout petit à nager. En guise de prière à nos bleus du ciel et de la mer. 